\title{
Sentiment Analysis Approaches and Applications: A Survey
}

\author{
Shamsa Umar \\ Department of Software \\ Engineering \\ The University of Lahore \\ Gujrat Campus, Gujrat
}

\author{
Maryam \\ Department of Software \\ Engineering \\ The University of Lahore \\ Gujrat Campus, Gujrat
}

\author{
Fizza Azhar \\ Department of Computer \\ Science and IT \\ University of Sialkot \\ Pakistan
}

\author{
Sayyam Malik \\ Department of Computer Science \\ The University of Lahore \\ Gujrat Campus, Gujrat
}

\author{
Ghulam Samdani \\ Department of Software Engineering \\ The University of Lahore \\ Gujrat Campus, Gujrat
}

\begin{abstract}
Nowadays utilization of social communication sites are developing various types and nature's of client are being use this benefits often times. Peoples on social media share their views, opinions and emotions symbolically or in text form. This situation and trend attract attention towards the research of sentiment analysis. Therefore, sentiment investigation idea is proposed. Among various applications of Natural Language processing (NLP) and Machine Learning (ML) Sentiment Analysis (SA) is very popular. The vital task of sentiment analysis is classification of sentiments, by automatically classifying the opinions/reviews and sentiments into three classes positive, negative and neutral. Many classification researches are conducted over the years to know the exact feelings and situations of sentimental emotions of peoples. Classification, fuzzy and clustering is used. Fuzzy based classification is finding more accurate. Furthermore the classical text classification models are utilized for comparative execution study. The comparative performance study shows the viability of the proposed order method and capable to deliver the more precise outcomes when contrasted with conventional classifiers. In this paper we have discussed different researcher's work on sentimental analysis approach and classification. This paper also presents the importance of opinion mining and sentiment analysis.
\end{abstract}

\section{Keywords}

Sentiment Analysis

\section{INTRODUCTION}

In this advanced era ,numerous people extensive use of internet and share their views, opinions, recommendations and self-experience about any specific product, politics and burning issues .However it is being hard to analyze the right selection about any domain according to suggestions and views of the people's. It's become difficult for the assessment of user's opinions weather negative or positive towards specific item for buying or examining[22] .In this situation investigators wish to declare sentimental analysis on this data set to find the polarity about specific item. So a computer biased machine can be invented to take these fast decisions in accurate way. The main thing is to extract the meaningful data and analyze the observation of user's to implement sentiment because mostly peoples use this way to find other's views for self satisfaction in opinion mining. However, some issues raised in opinion mining $(\mathrm{OM})$ like as few words are representing allegorical meaning in different perspective . For example the word "small" gives negative and positive sense according to special item if we use "this Smartphone is small" here this word gives positive view, in other side we say "the timing of the battery is small" this shows the negative perspective[23] In sentiment analysis classification of user written text is very crucial task. This classification is broadly consider for many levels [24]: document-level, [25] sentence-level [26] and aspect-level [27]. Document-level SA intends to arrange a feeling report as communicating a positive or negative conclusion or assessment. It considers the entire While Sentence-level SA plans to arrange sentiment communicated in every sentence. There are not such difference between document-level and sentence-level classification because sentences are just short documents. Document-level and Sentence-level classification does not provide the important detail required feelings on all parts of the substance which is required in numerous applications, to get these points of interest. So we need Aspect-level intends to group the sentiment as for the particular parts of elements. Very first step is to identify the aspect and entities because there are different opinions on the same entities. To perform the classification tasks for SA ,machine learning techniques and lexicon based strategies are used In few situation the hybrid approaches which merge lexicon base and machine learning approaches to be hired [28], [29].Furthermore lexicon based .Furthermore lexicon based sentiment analysis approach is composed of two sub categories i.e. dictionary base and corpus base. In order to cope with the challenges of large scale data, machine learning based approaches have been employed for sentiment analysis. These approaches are supervised learning, unsupervised and semi supervised learning based approaches/methods.

Supervised learning is based on existing trained document and show favorable outcomes. Support Vector Machine(SVM), Naïve Bayes(NB), Decision Tree(DT) ,Maximum Entropy(ME) and Neural Network (NN) are most useful algorithms in supervised learning NB is the most popular classifier has been used for sentiment analysis [30,31].

Unsupervised learning is useful for solving the difficulties in labeling the unlabeled data because this method is slighter depend and execute the superior result .many researchers use this strategy $[32,33]$. 
Semi-supervised techniques is difficult as compare to supervised and unsupervised [31] The idea behind the semi is that it contains knowledge about classes but hold information related to connected distribution on classification features [34]. If labeled data is limited then implementation on semi supervised approach is return outer performance as compare to other techniques.

In last years, many different techniques have been developed on sentimental analysis. The major tasks in sentimental analysis are subjectivity, lexicon base, opining mining, spam observation of opinion and superiority of inspection [35].The main purpose of this study is to discover the basic abstraction in the sector of Natural Language Processing and perform its comparative observation on sentimental analysis by using different techniques and approaches and declared that weather which strategy is best regarding to which approach and which techniques.

\section{RELATED WORK}

In this paper [1] the author described the study of emotional analysis by using micro blog sentimental analysis based on deep learning. The basic purpose of micro blog sentiment analysis is to find out the user's behavior and opinions about hot events. In this proposed methodology, the author's described a new technical solution. In this solution, the crawler crawled some data from the micro-blog. After the given corpus pretreatment, the Convolution Neural Network accepts as a sample of input and then two sentences emotion classifier is developed on the basis of SVM and RNN. In the final stage, the emotional analysis is fined. The practice proves that the proposed approach is more feasible to enhance the performance as compare to other techniques.

In [2] this survey article, the author's argues about scattered data on different platforms which is managed nowadays by using Machine learning and vocabulary based techniques to execute sentiment analysis and especially concentrate on four basic tasks respectively. In previous publication used many approaches and categories by comparing their results and accuracy but most founded are SVM and NB in this survey because approximation of these are high and cited for new machine learning techniques. At the end, the author has some provocations on the other languages due to their linguistic complexity and need to enhance other languages efficiency of machine learning techniques.

In this proposed article [3] the author elucidates the polarity of sentiment analysis on the scattered data by using SVM. In recent times, many features, classification and algorithm are proposed but no any mechanism is suited for each condition however, the author put forward Chi-square difference between the positive and negative categories(CDPNC) algorithm by merging $\mathrm{DF}$ and $\mathrm{CH}$, to establish the experimental supremacy of classification as compare to feature selection procedure and it produced good result .In the end the author analyze that the proposed strategy is adeptly good for sentence and document level but need to improve for word level sentiment.

In this paper [4] the author focus on utilizing Twitter, the most famous micro blogging stage, for the investigation of sentiment analysis. Author demonstrate to naturally gather a corpus for sentiment examination and opinion mining purposes. For this, use different techniques and tools like Tree Tagger for POS-tagging and observed the difference in distributions among positive, negative and neutral sets. Experimental assessments demonstrate that our proposed procedures are productive and performs superior to anything as compare to already proposed methods.

The proposed methodology [5] described that many Korean newspapers are showing bias due to their bias reporting. Writers of the daily paper article tend to express their aim indirectly that is, they pick a technique to forget some critical actualities, or now and then uses one-sided terms to bolster their assessment. It is difficult to assess their nature of writing. In this paper, we propose a strategy to identify political bias in the Korean articles by first building word vectors and sentence vectors, and second do a DBN-Training with those vectors and at long last do a relapse with SVM to compute the bias. We utilized our own particular dataset which is scored with the political bias some time recently doing the relapse.

After studding this article [6] Sentiment analysis of text content is vital part of seeing how person manage or understand text content and has the wide application in the industry. Having deep interest and successes of learning we proposed to work on sentiment analysis through deep learning models. In this paper, we propose a framework called Word2vec + Convolution Neural Network (CNN). Firstly, we utilize the word2 vec proposed by Google to register vector representations of words, which will be the contribution for the CNN. The motivation behind utilizing word2 $\mathrm{vec}$ is to pick up the vector representation of word and distance the separation of words. We also use the Parametric Rectified Linear Unit (PReLU), Normalization and Dropout technology to improve the accuracy and generalize ability of our model. Through this test we accomplished exactness of $45.4 \%$ in this dataset, which is a superior execution than some other neural system display like Recursive Neural Arrange (RNN) and Matrix-Vector Recursive Neural Network (MV-RNN).

In this research [7] during the most recent decade the Internet has been progressively utilized by individuals not just as clients but rather as procedures of the online web data. The themes of discussions on the forums were connected to health and fertility problems, fertility treatment and in vitro fertilization. The paper specially presents machine learning investigates order of assumptions in discussion writings. Current investigations had a few goals. The principle objective was to handle various explanations of posts; the second one was to think about a few feeling dictionaries and locate a superior one for these specific writing. In general, the results we reach the average F-measure equal to 0.805 .

The article [8] has conduct opinion mining on Roman Urdu because research work on other languages except English are very few, so the author has proposed sentiment on Roman Urdu by using classifications through the Waikato environment of knowledge analysis (WEKA).Hence to perform sentiment on Roman Urdu, the author has used three algorithms (KNN, Naïve Bayesian ,Decision Tree) . After applying and comparing these algorithms analyzed that Naive Bayesian algorithm performed more accurate task according to other algorithms.

Reference [9] described sentimental analysis by using domain adaptation algorithm on the bias of semi _ supervised approach to increase its performance . In this approach, views are categorized by applying maximum entropy(ME) and allot weight then bunch the categorized words through the point wise mutual information (PMI) .Domain adaptability is the main complication in sentimental analysis, however the author has labeled this in his proposed methodology and declare comparatively good result in comparison of baseline method. 
In this paper [10] the author has explored different strategies and approaches applied by other researchers and compare these procedures by introducing new concept of fuzzy classification for acquiring quality. The author has proposed fuzzy concept with new method which is useful for the strategy of text base. It shows high performance as compare to traditional techniques

In this[11] paper the author show SentiCircles, a lexiconbased approach for sentiment analysis with respect to Twitter. Unique in relation to run of the typical lexicon-based methodologies, which offer a fixed and static prior sentiment polarities of words paying little mind to their unique circumstance, Senti Circles takes into record the co-event examples of words in various settings in tweets to catch their semantics and upgrade their pre-assigned out quality and extremity in sentiment lexicons as needs be. The author recommends approach that considers the recognition of conclusion at both elements level and tweet-level. That's why in this paper[11] the author applied these proposed approach on three Twitter datasets utilizing three distinctive sentiment lexicons to determine word earlier sentiments. As result of these proposed approach, fundamentally outflanks the baselines in precision and F-measure for entity-level subjectivity. The proposed approach for tweet-level sentiment identification, performs superior to the state-of-the-art SentiStrength by $4-5 \%$ in precision in two datasets, however falls hardly behind by $1 \%$ in F-measure in the third dataset.

In this[12] paper the author explained the increasing interest because of the spread and development of social communities and networks. Specialists, researchers and decision makers confronts challenges for SA online surveys on the grounds that such comments are composed in unstructured arrangements with normally informal languages, expressions and conceivably mixed languages. Numerous challenges exist because of the Arabic language complexity and the predetermined number of research publications and datasets gathered and analyzed for such reason. Essentially two methodologies managed (corpus-based) and unsupervised (lexicon- based) decide the extremity of reviews. The author utilized the second approach and manufactures a huge sentiment lexicon and a lexicon-based SA tool. Thus the proposed approach appeared extraordinary outcomes, regarding expectation precision, contrasted with the keywordbased approach.

Number of individuals is utilizing online networking to express their suppositions on different subjects so this paper depicts a Twitter sentiment investigation framework that arranges a tweet as positive or negative in view of its general tweet-level extremity [13]. Administered learning classifiers frequently misclassify tweets containing conjunctions, for example, "but, and conditionals, for example, "if", because of their extraordinary semantic attributes. These classifiers likewise lot a choice score near the choice limit for an expansive number tweets, which recommends that they are essentially uncertain of being totally off-base about these tweets. To counter these two difficulties, this paper proposes a framework that improves administered learning for extremity characterization by utilizing on semantic principles and sentic computing resources. The author's proposed technique is assessed on two openly accessible Twitter corpora to delineate its adequacy [13].

In this paper, the author focus on utilizing Twitter, the most famous micro blogging stage, for the investigation of sentiment analysis [4]. The author demonstrates to naturally gather a corpus for sentiment examination and opinion mining purposes. For this the author use different techniques and tools like Tree Tagger for POS-tagging and observed the difference in distributions among positive, negative and neutral sets. Experimental assessments demonstrate that our proposed procedures are productive and performs superior to anything as compare to already proposed methods.

This paper, elaborate the issue of comprehension human sentiments from large scale online networking pictures, considering both visual substance and logical data, for example, remarks on the pictures, inscriptions, and so on. The challenge of this issue lies in the "semantic gap" between lowlevel visual elements also, larger amount picture opinions. Also, the absence of appropriate comments/marks in the dominant part of online networking pictures exhibits another challenge.a novel Unsupervised Sentiment Analysis (USEA) system for web-based social networking pictures is proposed to address these two difficulties. This approach exploits relations among visual substance and significant relevant data to connect the "semantic gap" in the forecast of picture sentiments. With experiments on two large-scale datasets, demonstrate the proposed technique is powerful in tending to the two difficulties[14].

Recently, Social media have turned into a developing wonder due to the enormous and rapid advances in information technology. Individuals are utilizing web-based social networking on everyday schedule to discuss their assessments with each other about wide assortment of subjects, items and administrations. There is a massive measure of client audits or sentiments created and shared every day. Twitter is a standout amongst the most generally utilized online networking micro blogging sites. Mining client suppositions from online networking data is not a straight forward errand; it can be achieved in various ways. The author utilizes, an open source approach, all through which, twitter Microblogs data has been gathered, pre-prepared, dissected and visualized utilizing open source devices to perform sentiment analysis and assumption examination for investigating client contributed online audits around two giant retail stores in the UK in particular Tesco and Asda stores over Christmas period 2014. Gathering client sentiments can be costly and time consuming task utilizing traditional techniques, for example, surveys. The sentiment analysis of the client assessments makes it simpler for organizations to comprehend their focused esteem in a changing business sector and to comprehend their client sees about their items and administrations, which additionally give knowledge into future promoting procedures and basic leadership arrangements[15].

An imperative contrast between traditional AI frameworks and human knowledge is the human capacity to outfit realistic information gathered from a lifetime of learning and experience to settle on educated choices. This permits people to adjust effortlessly to novel circumstances where AI comes up short disastrously because of an absence of situationspecific standards and speculation capacities. Commonsense learning additionally gives foundation information that empowers people to effectively work in social circumstances where such learning is commonly expected. Since commonsense comprises of information that people underestimate, gathering it is an amazingly troublesome undertaking. In this work, the author utilized a group of hierarchical clustering and dimensionality lessening for naturally finding the primitives for both noun and verb ideas in SenticNet [16]. This generalization handle permitted to generally broaden the scope of the commonsense knowledge base. Past forms of SenticNet were centered on gathering this 
sort of learning for sentiment analysis yet they were vigorously restricted by their failure to sum up SenticNet 4 overcomes such confinements by utilizing on conceptual primitives consequently created by method for hierarchical clustering and dimensionality reduction.. This paper proposed a novel technique to anticipate the feelings and group progression by examining the code review comments [17]. The code review from source control vault was removed for improvement movement from the previous three years for two open source ventures. The comments were tokenized and elements were extricated to recognize sentiments, for example, consolation, inconvenience and dread. The feeling extremity of the code review comments were measure as far as neutral vs. rude (harshness). The strategy utilized gave an exactness of $67 \%$ regarding acknowledgment of harsh comments.

This paper depicts a strategy to distinguish transient connections between news articles utilizing phrase position investigation. The words from the news feed were tokenized utilizing extraordinary character and space delimiters. The word-tokens were labeled by the sort, for example, noun, verb, preposition and adverb. The area of every word and its separation from the verb on the left, right and past and next sentence was recorded as a component. The connections between various sentences utilizing nouns as the anchor and verbs as the occasion were set up. The connection check was measured and the associated chart was put away as a choice tree. The model was tried utilizing temporal questions and event sequence queries. The consequence of proposed system showed $67 \%$ exactness regarding right event sequencing and recall rate. Also feeling based classification of the news occasion was performed to test whether the weighted diagram connecting in view of expression position strategy could be utilized for sentiment analysis with dread and satisfaction order demonstrating clear edges for certain occasion combine and their temporal relation type [18]

Stock price forecasting is imperative in the arranging of business activity. The objective of this research is to assemble a model to foresee stock price development utilizing the sentiment from online networking. Not at all like past methodologies where the general mind-sets or assessments are considered, had the sentiments of the particular points of the organization consolidated into the stock expectation display. Themes and related sentiments are naturally separated from the texts in a message load up by utilizing the proposed strategy and in addition existing subject models. In addition, this paper demonstrates an assessment of the adequacy of the sentiment analysis in the stock expectation undertaking by means of a large scale test. Looking at the exactness average more than 18 stocks in one year transaction, the author proposed technique accomplished $2.07 \%$ better execution than the model utilizing historical costs only .Furtherrmore, when contrasting the strategies just for the stocks that are hard to predict, this strategy accomplished $9.83 \%$ better precision than historical cost method, and $3.03 \%$ better than human opinion strategy [19].

This study concentrates on aspect-level sentiment analysis, where the objective is to discover and aggregate sentiment on entities mentioned within documents or aspects of them. A top to bottom outline of the current state-of-the-art is given, demonstrating the huge advance that has as of now been made in finding both the objective, which can be an element thusly, or some part of it, and the relating sentiment. Aspect-level sentiment analysis yields exceptionally fine grained sentiment data which can be helpful for applications in different spaces.
Current arrangements are ordered based on whether they give a strategy to aspect detection, sentiment analysis, or both. Moreover, a breakdown in view of the sort of algorithm utilized is given. For each discussed study, the revealed execution is incorporated. To encourage the quantitative assessment of the different proposed techniques, a call is made for the institutionalization of the assessment strategy that incorporates the utilization of shared information sets. Semantically-rich concept-centric aspect-level assessment examination is talked about and distinguished as one of the most encouraging future research course [20].

Sentiment Analysis (SA) or opinion mining(OM) has as of late turned into the concentration of numerous analysts, since examination of online content is gainful and requested for statistical surveying, logical studies from psychological and sociological point of view, political surveys, business insight, upgrade of web based shopping frameworks, and so forth. These days on the off chance that one needs to purchase a consumer item one lean toward client reviews and examination out in the open discussions on web about the item. Subsequently opinion mining has picked up significance. This online verbal speaks to new and quantifiable wellspring of data with numerous applications, this procedure of distinguishing and separating subjective data from crude information is known as sentiment analysis. The author discussed different sentiment classification techniques and tools for sentiment classification. This paper also displays a study on assessment examination or opinion mining [21].

\section{INITIATIVE PROCEDURE OF SENTIMENT ANALYSIS}

Numerous use of internet sentimental analysis subjectivity analysis and opinion mining are similar areas of research that are using different techniques derived from NLP, structured and un structured data mining and information retrieval(IT).sentimental is a multi lingual problem not a single [36]. Many researcher's struggle have been seen in most early years that help to automated the sentiment analysis and the author extend the research of NLP [37]. For the sentiment of data ,data procurement and preprocessing of data are most common steps .Data procurement and data preprocessing are must needed in the field of sentiment analysis. The concept of sentiment is given by the Nusukawa and yi in 2003 and opinion mining concept is given by the Dave ,Lawrence in 2003. In [38,39,40] carried out the study on the polarity through the Machine learning $[41,42,43]$ lexicon based and Hybrid based [44,45]. In [46] the author focused on specific problems that are occurring in SA and OM fields so they proposed classification levels(Document Level, Sentence Level, Aspect Level, Comparative Sentiment Analysis ,Lexicon Acquisition ) for various specific rising problems.

\section{SENTIMENT ANALYSIS LEVELS} 4.1 Document Level

Is performed for the entire report and afterward choose whether the document express positive or negative assumption. The essential data unit is a solitary archive of obstinate content. In this document level characterization, a solitary audit about a solitary point is considered. Be that as it may, on account of discussions or online journals, similar sentences may show up. Clients may contrast one item and another that has comparative attributes and thus record level investigation is not alluring in gatherings and web journals. There are two principle ways to deal with document level sentiment examination: administered learning and 
unsupervised learning. The administered approach expect that there is a limited arrangement of classes into which the archive ought to be grouped what's more, preparing information is accessible for every class. The least difficult case is at the point when there are two classes: positive furthermore, negative. Straightforward expansions can likewise include an unbiased class or have a few discrete numeric scales into which the archive ought to be set (like the five-star framework utilized by Amazon). Given the preparation information, the framework takes in a grouping model by utilizing one of the regular grouping calculations, for example, SVM, Naive Bayes, Strategic Regression, or KNN. This grouping is then used to tag new records into their different estimation classes. At the point when a numeric esteem (in some limited range) is to be allotted to the record then relapse can be utilized to anticipate the incentive to be allocated to the report (for instance, in the Amazon five-star positioning framework). Look into this has demonstrated that great exactness is accomplished not withstanding when each report is spoken to as a straightforward pack of words [47]. There are also two main approaches to the selection of the phrases: a set of predefined POS patterns can be used to select these phrases or a lexicon of sentiment words and phrases can be used [48]. In this manner the document level opinion grouping has its own particular points of interest and drawbacks. Favorable position is that we get a general extremity of sentiment content about a specific substance from a record. Disservice is that the distinctive feelings about various elements of an element couldn't be removed independently[49].

\subsection{Sentence Level}

A solitary record may contain various suppositions even about the same elements. When we need to have an all the more fine-grained perspective of the distinctive suppositions communicated in the document about the elements we should move to the sentence level. In the sentence level slant investigation, the extremity (positive/negative/nonpartisan) of every sentence is ascertained. A similar report level characterization strategies can be connected to the sentence level arrangement issue. Objective and subjective sentences must be discovered. The subjective sentences contain assessment words which help in deciding the slant about the substance. After which the extremity characterization is done into positive and negative classes. Sentence level slant grouping is not alluring in the event that mind boggling sentences, it is helpful if there should arise an occurrence of single and straightforward sentence. Most strategies utilize supervised approaches to deal with order the sentences into the two classes [50]. A bootstrapping methodology was recommended by Hai keeping in mind the end goal to decrease the measure of physical work required while setting up a substantial preparing corpus [51]. An extraordinary approach in view of the base cuts was proposed in Pang and Lee [52]. The fundamental preface of their approach is that neighboring sentences ought to have a similar subjectivity characterization. As specified before, generally approaches to sentence-level assessment examination are either in light of directed learning or on unsupervised learning [53]. The last approach is comparable in nature to that of Turney, with the exception of that it utilizes an altered log-probability proportion rather than PMI and the quantity of seed words that are utilized to discover the SO of the words in the sentence is much bigger [50].

\subsection{Aspect Level}

The two past methodologies function admirably when either the entire record then again every individual sentence alludes to a solitary element. Aspect-based sentiment analysis (also called feature-based sentiment analysis) is the exploration issue that spotlights on the acknowledgment of all sentiment expressions inside a given report and the angles to which they allude. The aspect level assumption grouping is an a great deal more pinpointed way to deal with conclusion mining. The expressions that contain supposition words are discovered and an expression level characterization is finished. This can be beneficial or disadvantageous. The classical approach, which is utilized by numerous business organizations, to the recognizable proof of all perspectives in a corpus of item surveys is to concentrate all thing phrases (NPs) and afterward keep only the NPs whose recurrence is over some tentatively decided threshold [54]. One approach is to decrease the commotion in the discovered NPs Now and again, the correct feeling around a substance can be effectively removed [55]. Be that as it may, in some different cases, where relevant extremity additionally matters, the outcome may not be completely exact. Another way to deal with viewpoint recognizable proof is to utilize an expression reliance parser that uses known assessment expressions to discover extra angles (indeed, even occasional ones) [56]. We can likewise see the issue of viewpoint ID as a data extraction issue and after that utilization a labeled corpus to prepare a succession classifier for example, a Conditional Random Field (CRF) to discover the aspects [57]. Invalidation of words can happen locally. In such cases, this level of sentimental analysis suffices. In any case, if there are sentences with invalidating words which are far separated from the supposition words, state level investigation is not desirable. The words that seem exceptionally close to each other are thought to be in an expression. The last extremity of every viewpoint is controlled by a weighted normal of the polarities of all notion expressions contrarily weighted by the separate between the perspective and the assessment expression [58].

\section{SENTIMENT ANALYSIS TECHNIQUES}

To perform sentiment analysis there are two main techniques machine learning approach and lexicon based approach. The next one is Hybrid approach. This approach is the combination of Machine learning and Lexicon based techniques. Machine learning approach is used to perform the task of sentimental analysis on famous Machine learning approach on the text data. Machine learning approach can be further divided into supervised and unsupervised classification. The lexicon based approach is based on lexicon. Lexicon based approach is further divided into sub tasks Dictionary based and Corpus based [59,60,61].

\subsection{Lexicon Based Approach}

In this unsupervised techniques the classification is held on features based it is consider polarity of people's views and opinions and then analyze that it is more positive or it is negative , that's called sentiment lexicon. For compiling the sentiment word net used manually approach but it's tough and time taking process and do not perform any task alone. Its needed further two automated approaches that perform automatically sentiment these two automated approaches are defined below $[59,60,61]$.

\subsection{Dictionary Based Approach}

This present the basic policy of dictionary based techniques [62], [63]. It first identify the polarity of the word after that 
find it in dictionary and its synonym and antonym and known as collection WorldNet [64], [65], [66] or word finder [67].Its follow the iterative process after finding new word then add it in stem words. Repeat this iterative process until not search new words. Then manually inspections continue for error correction. In [68] the author has used this methodology for adjectives of 30 adjectives because it has limitations so he observed that it cannot search opinion words. In this approach has a big drawback that it not fined the opinion words about specific domain [69]. The next author has proposed rule based approach in to over this issue and fined good performance in the extraction of the ad selection and advertising keywords [70].

\subsection{Corpus Based Approach}

Corpus based approach relatively perform good accuracy by solving the problem of finding specific opinion words with specific orientations, this is the big advantage of Corpus based approach which don't have in dictionary based approach. IN the research[71] the author has used the Conditional Random Fields (CRFs) method for opinion extractions through the manually learning techniques. This was also used by the author [72] to identify multi string polarity pattern matching algorithm that was used on the reviews of Chinese. They work on different items and produced good result. The next author [73] used two levels CRF model to bring out relative relations without interdependencies. This shows the result as compare to other models are relatively good and proved that its effective tool. The single use of the corpus based approach is not impressive as compare to Dictionary based approach because it can't handle big corpus but it is useful for specific opinion words and its orientation. This approach is utilized through the statistically approach or semantic approach.

\subsubsection{Statistical Approach}

is used to search co-occurrence patterns and stem the opinion words in this technique. This is used for many related application to Sentiment analysis. For finding the polarity of big annotated word corpus [74].

\subsubsection{Semantic Approach}

is rely on principle to analyze the similar calculation to show its semantic value to directly. This is used in different applications to make lexicon base model for the expressing of Noun, verbs, Adjective and adverbs are used in sentiment analysis as the author has used in [75].In this author has use both methods to produce the deficiencies of people's online review in sentiment analysis and utilize the feature base and sentence based. They present the high performance in finding weakness [76].

\subsection{Hybrid Approach}

The combination of Machine learning and lexicon based approach is called the Hybrid approach. Some research techniques have analyze that hybrid approach improves the performance in sentiment classification. In this author has used these both techniques together in concept level sentiment analysis and perform high accuracy [77].

\section{CONCLUSION}

With the expand use of internet, in this survey we declared the significance of opinion mining and sentimental analysis .Sentimental analysis is developing zone as individuals are sharing their perspectives, opinions and encounter on the web. people share their views and comments in various fields ,to extract the meaning full information about specific field various researchers elucidate different methodology by using different techniques ,applications ,tools and Algorithms. In this paper we summarize the current research and review the related work and declared results after studying the Techniques, Approaches and Classification .We analyze through the observation of publication that sentiment analysis issues can solve by using various approaches ,tools and Algorithm according to its features perspective regarding to its good performance. But still there is no introduced that proper techniques which handle all the challenges. After the survey of some researcher's work we analyze that support vector machine (SVM) is presenting high performance as compare to other algorithms but it also not fully perform good but having some limitations. In future we need to high performance in sentiment analysis because in this advance era industries demand such a perfect application that analyze and feel the user's wishes and their competitors . combine different techniques to overcome the drawbacks and advantages of each individual's merits and increase the performance of sentiments analysis.

\section{REFERENCES}

[1] L. Yanmei and C. Yuda, "Research on Chinese MicroBlog Sentiment Analysis Based on Deep Learning," 2015 8th Int. Symp. Comput. Intell. Des., pp. 358-361, 2015.

[2] E. Aydogan and M. A. Akcayol, "A comprehensive survey for sentiment analysis tasks using machine learning techniques,” 2016 Int. Symp. Innov. Intell. Syst. Appl., pp. 1-7, 2016.

[3] F. Luo, C. Li, and Z. Cao, "Affective-feature-based sentiment analysis using SVM classifier," 2016 IEEE 20th Int. Conf. Comput. Support. Coop. Work Des., pp. 276-281, 2016.

[4] A. Pak and P. Paroubek, "Twitter as a Corpus for Sentiment Analysis and Opinion Mining," pp. 13201326.

[5] T. Mikolov, K. Chen, G. Corrado, and J. Dean, "Efficient Estimation of Word Representations in Vector Space," Arxiv, no. 9, pp. 1-12, 2013.

[6] X. Ouyang, P. Zhou, C. H. Li, and L. Liu, "Sentiment Analysis Using Convolutional Neural Network," Comput. Inf. Technol. Ubiquitous Comput. Commun. Dependable, Auton. Secur. Comput. Pervasive Intell. Comput. (CIT/IUCC/DASC/PICOM), 2015 IEEE Int. Conf., pp. 2359-2364, 2015.

[7] V. Bobicev, "Text classification: The case of multiple labels,” IEEE Int. Conf. Commun., vol. 2016-Augus, pp. 39-42, 2016.

[8] M. Bilal, H. Israr, M. Shahid, and A. Khan, "Sentiment classification of Roman-Urdu opinions using Na??ve Bayesian, Decision Tree and KNN classification techniques,” J. King Saud Univ. - Comput. Inf. Sci., vol. 28, no. 3, pp. 330-344, 2016.

[9] J. S. Deshmukh, "Mining Multi Domain Text Reviews Using Semi- Supervised Approach," no. March, 2016.

[10] V. Bairagi and N. Tapaswi, "Social network comment classification using fuzzy based classifier technique," 2016 Symp. Colossal Data Anal. Networking, CDAN 2016, 2016

[11] H. Saif, Y. He, M. Fernandez, and H. Alani, "Contextual Semantics for Sentiment Analysis of Twitter." 
[12] S. N. Mining, "Lexicon-based sentiment analysis of Arabic tweets Mahmoud Al-Ayyoub* and Safa Bani Essa Izzat Alsmadi," vol. X.

[13] P. Chikersal, S. Poria, E. Cambria, A. Gelbukh, and C. E. Siong, "Modelling Public Sentiment in Twitter: Using Linguistic Patterns to Enhance Supervised Learning," pp. 49-65.

[14] Y. Wang, S. Wang, J. Tang, H. Liu, and B. Li, "Unsupervised Sentiment Analysis for Social Media Images."

[15] E. M. G. Younis, "Sentiment Analysis and Text Mining for Social Media Microblogs using Open Source Tools : An Empirical Study Sentiment Analysis and Text Mining for Social Media Microblogs using Open Source Tools : An Empirical Study," no. February, 2015.

[16] E. Cambria, S. Poria, and R. Bajpai, "SenticNet 4 : A Semantic Resource for Sentiment Analysis Based on Conceptual Primitives," pp. 2666-2677, 2016.

[17] A. Veenendaal et al., "Sentiment Analysis in Code Review Comments," vol. 4, no. 6, pp. 2014-2015, 2016.

[18] A. Veenendaal, E. Jones, Z. Gang, E. Daly, S. Vartak, and R. Patwardhan, "Temporal Analysis of News Feeds using Phrase Position," pp. 2-7.

[19] T. Hai, K. Shirai, and J. Velcin, "Sentiment analysis on social media for stock movement prediction," Expert Syst. Appl., vol. 42, no. 24, pp. 9603-9611, 2015.

[20] K. Schouten and F. Frasincar, "Survey on Aspect-Level Sentiment Analysis," pp. 1-20.

[21] S. K. Yadav, "Sentiment Analysis and Classification: A Survey," pp. 113-121, 2015.

[22] Khushboo, Trivedi N., Swati K. Vekariya, and Shailendra Mishra. "Mining of sentence level opinion using supervised term weighted approach of Naïve Bayesian algorithm." International Journal of Computer Technology and Applications 3.3 (2012).

[23] Rashid, Ayesha, et al. "A survey paper: areas, techniques and challenges of opinion mining." IJCSI International Journal of Computer Science Issues 10.2 (2013): 18-31.

[24] Thomas, B., 2013. What Consumers Think About Brands on Social Media, and What Bunesses Need to do about it Report, Keep Social Honest?

[25] Yessenalina, Ainur, Yisong Yue, and Claire Cardie. "Multi-level structured models for document-level sentiment classification." Proceedings of the 2010 Conference on Empirical Methods in Natural Language Processing. Association for Computational Linguistics, 2010.

[26] Farra, Noura, et al. "Sentence-level and document-level sentiment mining for arabic texts." Data Mining Workshops (ICDMW), 2010 IEEE International Conference on. IEEE, 2010. [26]

[27] Zhou, Haochen. Aspect-Level Sentiment Analysis Based on a Generalized Probabilistic Topic and Syntax Model. Diss. 2014.

[28] Z. Nanli, Z. Ping, L. Weiguo, and C. Meng, "Sentiment analysis: A literature review" International Symposium on Management of Technology (ISMOT), pp. 572-576, 2012 .
[29] W. Medhat, A. Hassan, H. Korashy, "Sentiment analysis algorithms and applications: A survey", Ain Shams Engineering Journal, vol. 5, no. 4, pp. 1093-1113, 2014.

[30] B. Florian, F. Schultze, and L. Strauch, "Semantic Search: Sentiment Analysis with Machine Learning Algorithms on German News Articles", 2015.

[31] M.-T. Martín-Valdivia, E. Martínez-Cámara, J.-M. Perea-Ortega, and L.A. Ureña-López, "Sentiment polarity detection in Spanish reviews combining supervised and unsupervised approaches", Expert Systems with App?ications, vol. 40, no. 10, pp. 3934 3942, 2013

[32] H. Ghorbel and D. Jacot, "Sentiment Analysis of French Movie Reviews", In Advances in Distributed AgentBased Retrieval Tools, Springer Berlin Heidelberg, pp. 97-108, 2011.

[33] H. Nizam and S. S. Ak?n, "Sosyal Medyada Makine renmesi ile Duygu Analizinde Dengeli ve Dengesiz Veri Setlerinin Performanslar[n] Kar? 国la?t?

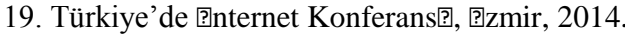

[34] K. Ravi and V. Ravi, "A survey on opinion mining and sentiment analysis: Tasks, approaches and applications", Knowledge-Based Systems, vol. 89, pp. 14-46, 2015.

[35] Liu, Bing. "Sentiment analysis and opinion mining." Synthesis lectures on human language technologies 5.1 (2012): 1-167.

[36] B. Liu, Sentiment analysis: A multi-faceted problem, IEEE Intelligent Systems 25, no. 3 (2010): 76-80. [12] S. N. Mining, "Lexicon-based sentiment analysis of Arabic tweets Mahmoud Al-Ayyoub * and Safa Bani Essa Izzat Alsmadi," vol. X.

[37] S. Poria, A. Gelbukh, A. Hussain, N. Howard, D. Das, S. Bandyo padhyay, Enhanced SenticNet with Affective Labels for Concept-Based Opinion Mining, KnowledgeBased Approaches to Concept-Level Sentiment Analysis, IEEE Intelligent Systems, (2013) 1

[38] L.K.W. Tan, J.C. Na, Y.L. Theng et al. Phrase-level sentiment polarity classification using rule-based typed dependencies and additional complex phrases consideration. Journal of Computer Science and Technology 27(3): 650-666 May 2012. DOI 10.1007/s11390-012-1251-y.

[39] Z. Zhang, Q. Ye, Z. Zhang, Y. Li, Sentiment classification of Internet restaurant reviews written in Cantonese, Expert Systems with Applications 38 (2011) 7674-7682.

[40] X. Bai, Predicting consumer sentiments from online text, Decision Support Systems 50 (2011) 732-742.

[41]T. Wilson, J. Wiebe, P. Hoffmann, Recognizing contextual polarity in phrase-level sentiment analysis, Proceedings of HLT/EMNLP-05, Vancouver, Canada, 2005.

[42] P. Turney, Thumbs up or thumbs down? Semantic orientation applied to unsupervised classification of reviews, In: Proceedings of the 40th annual meeting on association for computational linguistics ACL'02 (pp. 417-424), Stroudsburg, PA, USA: Association for Computational Linguistics, 2002. 
[43]Y. Lu, X. Kong, X. Quan, W. Liu, and Y. Xu, Exploring the Sentiment Strength of User Reviews, L. Chen et al. (Eds.): WAIM 2010, LNCS 6184, pp. 471-482, 2010.

[44] A. Balahur, Jesús M. Hermida, A. Montoyo, Detecting implicit expressions of emotion in text: A comparative analysis, Decision Support Systems 53 (2012) 742-753.

[45] M. Abdul-Mageed, M. Diab, S. Kübler, SAMAR: Subjectivity and sentiment analysis for Arabic social media, Computer Speech and Language 28 (2014) 2037.

[46] R. Feldman, Techniques and Applications for Sentiment Analysis, Review Articles, Communications of the ACM, Vol. 56 No. 4, Pages 82-89, April 2013.

[47] Pang, B., Lee, L. and Vaithyanathan, S. Thumbs up? Sentiment Classification using machine learning techniques. In Proceedings of EMNLP-02, 7thConference on Empirical Methods in Natural Language Processing (Philadelphia, PA, 2002). Association forComputational Linguistics, Morristown, NJ, 79-86.

[48] T urney, P. Thumbs up or thumbs down? Semantic orientation applied to unsupervised classification of reviews. In Proceedings of the Association forComputational Linguistics (2002), 417-424.

[49] T aboada, M., J. Brooke, J., Tofiloski, M., Voll, K. and Stede, M. Lexicon-based methods for sentiment analysis. Computational Linguistics 37, 2 (2011),267-307.

[50]Y u, H. and Hatzivassiloglou, V. Towards answering opinion questions: Separating facts from opinions and identifying the polarity of opinion sentences. InProceedings of the Conference on Empirical Methodsin Natural Language Processing (2003).

[51] R iloff, E. and Wiebe, J. Learning extraction patterns for subjective eExpressions. In Proceedings of theConference on Empirical Methods in Natural LanguageProcessing (2003).

[52] Pang, B. and Lee, L. A Sentimental Education: Sentiment Analysis using Subjectivity Summarization based on minimum cuts. In Proceedings of theAssociation for Computational Linguistics (2004), 271-278.

[53] Kim, S.-M. AndHovy, E. Crystal: Analyzing predictive opinions on the Web. In Proceedings of the JointConference on Empirical Methods in Natural LanguageProcessing and Computational Natural Language Learning (2007).

[54] $\mathrm{H} \mathrm{u}, \mathrm{M}$. and Liu, B. Mining and summarizing customerreviews. In Proceedings of the ACM SIGKDDConference on Knowledge Discovery and Data Mining(2004), 168-177.

[55] Popescu, A.-M. andEtzioni, O. Extracting product features and opinions from reviews. In Proceedings ofConference on Empirical Methods in Natural LanguageProcessing (2005).

[56] Wu, Y., Zhang, Q. Huang, $\mathrm{X}$. and $\mathrm{Wu}, \mathrm{L}$. Phrasedependency parsing for opinion mining. In Proceedingsof Conference on Empirical Methods in NaturalLanguage Processing (2009).

[57] Lafferty, J., McCallum, A. and Pereira, F. Conditionalrandom fields: Probabilistic models for segmenting and labeling sequence data. In Proc. 18th InternationalConf. on Machine Learning.Morgan Kaufmann, San Francisco, CA, 2001, 282-289.

[58] J akob, N. and Gurevych, I. Extracting opinion targetsin a single-and cross-domain setting with conditional random fields. In Proceedings of Conference onEmpirical Methods in Natural Language Processing(2010).

[59] W. Medhat, A. Hassan, H. Korashy, "Sentiment analysis algorithms and applications: A survey", Ain Shams Engineering Journal, vol. 5, no. 4, pp. 1093-1113, 2014.

[60] Diana Maynard, Adam Funk. Automatic detection of political opinions in tweets. In: Proceedings of the 8th international conference on the semantic web, ESWC'11; 2011. p. 88-99.

[61] W. Medhat et al. Sentiment analysis algorithms and applications: A survey, Ain Shams Eng J (2014), http://dx.doi.org/10.1016/j.asej.2014.04.011।.

[62] $\mathrm{Hu}$ Minging, Liu Bing. Mining and summarizing customer reviews. In: Proceedings of ACM SIGKDD international conference on Knowledge Discovery and Data Mining (KDD’04) 2004.

[63] Kim S, Hovy E. Determining the sentiment of opinions. In:Proceedings of interntional conference on Computational Linguistics (COLING’04); 2004.

[64] Miller G, Beckwith R, Fellbaum C, Gross D, Miller K.WordNet: an on-line lexical database. Oxford Univ. Press; 1990.

[65] M. Kanakaraj, R. Mohana, and R. Guddeti, "NLP Based Sentiment Analysis on Twitter Data Using Ensemble Classifiers,"in 3rd International Confrence on Signal Processing, Communication and Networking(ICSCN), 2015.

[66] S. Gao, J Hao, Y Fu, "The Application and Comparison of Web Services for Sentiment Analysis in Tourism."

[67] Mohammad S, Dunne C, Dorr B. Generating highcoverage semantic orientation lexicons from overly marked words and a thesaurus. In: Proceedings of the conference on Empirical Methods in Natural Language Processing (EMNLP'09); 2009.

[68] M. Hu and B. Liu, "Mining and summarizing customer reviews," Proceedings of the tenth ACM international conference on Knowledge discovery and data mining, Seattle, 2004, pp. 168-177.

[69] B. Liu, Web Data Mining: Exploring Hyperlinks, Contents, and Usage Data. Springer, 2006.

[70] Qiu Guang, He Xiaofei, Zhang Feng, Shi Yuan, Bu Jiajun, Chen Chun. DASA: dissatisfaction-oriented advertising based on sentiment analysis. Expert Syst Appl 2010;37:6182-91.

[71] Lafferty J, McCallum A, Pereira F. Conditional random fields:probabilistic models for segmenting and labeling sequence data. In: Proceedings of International Conference on Machine Learning (ICML'01); 2001.

[72] Jiao Jian, Zhou Yanquan. Sentiment Polarity Analysis based multi-dictionary. In: Presented at the 2011 International Conference on Physics Science and Technology (ICPST'11); 2011. 
[73] Xu Kaiquan, Liao Stephen Shaoyi, Li Jiexun, Song Yuxia Mining comparative opinions from customer reviews for competitive intelligence. Decis Support Syst 2011;50:743-54.

[74] Read J, Carroll J. Weakly supervised techniques for domain independent sentiment classification. In: Proceeding of the 1st international CIKM workshop on topic-sentiment analysis for mass opinion; 2009. p. 4552.

[75] Maks Isa, Vossen Piek. A lexicon model for deep sentiment analysis and opinion mining applications. Decis Support Syst 2012;53:680-8.
[76] Zhang Wenhao, Hua Xu, Wan Wei. Weakness finder: find product weakness from Chinese reviews by using aspects based sentiment analysis. Expert Syst Appl 2012;39:10283-91.

[77] A. Mudinas, D. Zhang, M. Levene, "Combining lexicon and learning based approaches for conceptlevel sentiment analysis", Proceedings of the First International Workshop on Issues of Sentiment Discovery and Opinion Mining, ACM, New York, NY, USA, Article 5, pp. 1-8, 2012. 